\title{
Virtual SEM (VSEM) - A Teaching And Training Solution For The SEM
}

\author{
Mr. G.C. Martin, Mr. B.C. Breton, Dr. N.H.M. Caldwell, Dr. D.M. Holburn. \\ Department of Engineering, University of Cambridge, Cambridge, United Kingdom, CB2 1PZ.
}

VSEM (Virtual Scanning Electron Microscope) is an Internet-based software suite for training new SEM users and teaching students about the instrument. The concept incorporates a simulator of the imaging operations of a real SEM, an encyclopaedia of SEM-related scientific and operational theory, and a computer-based learning $(\mathrm{CBL})$ module to guide training and learning.

It addresses three issues in the learning of modern Scanning Electron Microscopy, identified during the course of our earlier reported work [4]:

- Modern computer-control has removed the user from the SEM's "nuts and bolts". Today's operator therefore often lacks an in-depth knowledge of the fundamentals of SEM science, resulting in longer training times and potentially lower quality final images;

- Heavy demands on instruments and instructors frequently reduce available training time;

- School or university students often need an understanding of SEM science and capabilities. However, it can be expensive or impractical to make real microscopes available.

VSEM tackles each of these areas by reproducing the functionality of the SEM, and providing guided theoretical teaching. It can relieve the pressure on real instruments for training, enable students to gain a better appreciation of the microscope than would otherwise be possible, and provide a resource to develop new users' understanding of SEM science.

To date, research has concentrated on the development of the simulator (FIG. 1.) and encyclopaedia (with demonstration versions of these now being available [6]). The software is based entirely on Internet technologies - early in the project it was decided that, due to the variety of target environments for VSEM, it should be delivered via a web-browser to remove compatibility issues and simplify distribution. The encyclopaedia is written with Macromedia Flash and XML, while the simulation is a Java applet. Java will also be used for the computer-based assessment module. By embedding these different technologies into a HTML web page, it is possible to make them perform with the appearance of a single software package.

Several software simulations exist to demonstrate features of SEM operation (for example [1], [2], [3]). These often use digital video sequences to show the microscope image as a single control is adjusted. While elegant, this approach is impractical for fully simulating the functionality of the SEM - an overwhelming number of images are required to represent every possible control combination. Our simulator therefore employs digital image filtering to maximise realism.

Convolution of an original image with suitable kernels allows the reproduction of effects such as changing focus, brightness, contrast and astigmatism. Displaying only a portion of a large picture allows simulated magnification and panning. The great advantage of digital image filtering over video is that only a single sample image is required to show the cumulative effects of different operations. However, it also requires significantly more computer processing power to achieve. 
A variety of convolution techniques exist. The most commonly used are spatial domain convolution, and frequency domain convolution via Fast Fourier Transforms (FFTs) [5]. For small or simple kernels, the spatial domain offers the fastest, most efficient algorithms. However, as the kernel becomes more complex, frequency domain convolution quickly becomes the faster option, with the added attraction that it offers unlimited scope for the range of filters that may be applied.

The aim is to carry out the convolution in real-time, responding directly to user manipulation of the simulated controls. Our initial version of the simulator used spatial domain filtering to reproduce focusing, astigmatism, brightness, contrast and magnification. However, we have subsequently demonstrated the use of real-time filtering using frequency domain convolution, allowing the development of better software that behaves more realistically.

Planned future work will enable the CBL module in VSEM to set a trainee a task in the simulator. It will then assess the user's ability to operate the SEM to complete that task. For the purposes of teaching SEM science, the simulator could also be used to demonstrate a particular microscope function. Such example scenarios highlight the potential usefulness of VSEM, which will be assessed more thoroughly through forthcoming field-testing in the University of Cambridge.

\section{References:}

[1] FESEM simulator. http://www.vcbio.science.ru.nl/eng/fesem/applets/

[2] I. Lapsker and J. Azoulay, Microsc. Microanal. 9 (suppl. 2) (2003) 1262.

[3] Molecular Expressions Virtual SEM. http://micro.magnet.fsu.edu/primer/java/electronmicroscopy/magnifyl/index.html

[4] N.H.M. Caldwell et al., Microsc. Microanal. 8 (suppl. 2) (2002) 1566.

[5] R.C. Gonzalez and R.E. Woods, Digital Image Processing, Addison-Wesley, 1992.

[6] Virtual SEM Project Website. http://www.virtualsem.com

[7] G.C. Martin is supported by an EPSRC Studentship, through the Department of Engineering, University of Cambridge, UK.

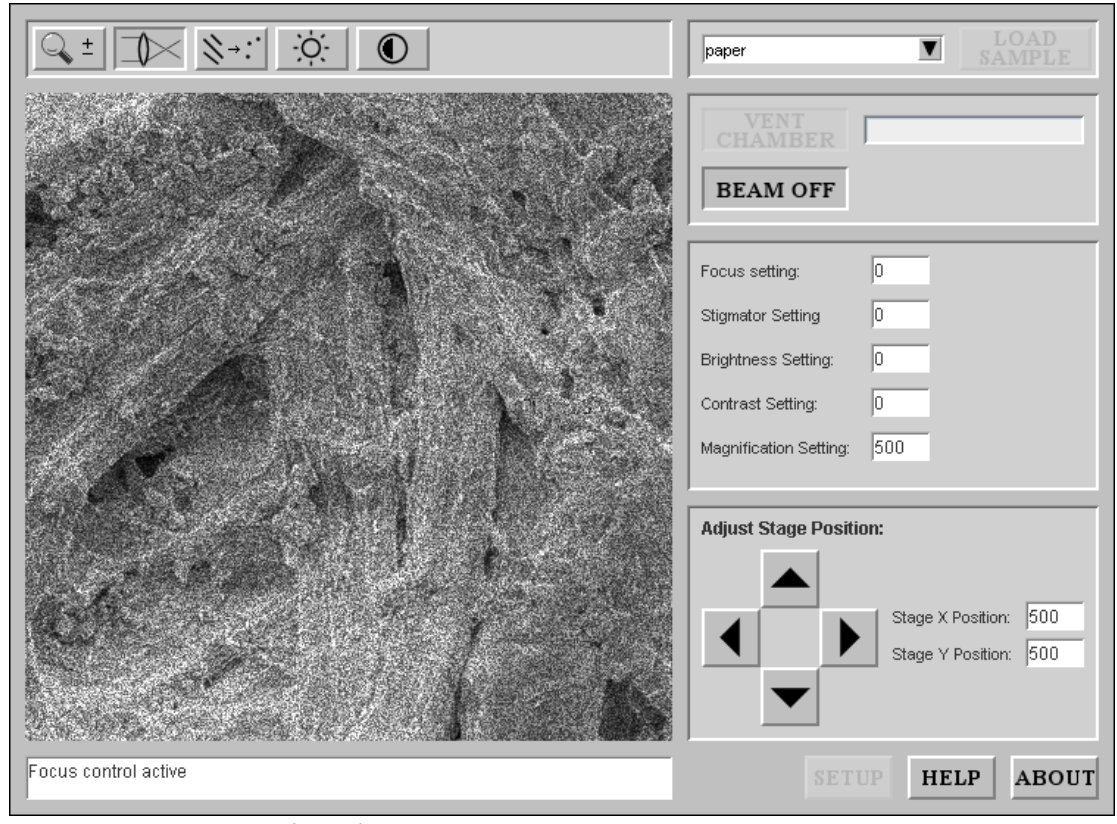

FIG. 1. VSEM Simulator 